\title{
The Asymmetrical Contributions of Pleasure and Pain To Animal Welfare
}

\author{
Adam Shriver \\ Ph.D. Student \\ Philosophy-Neuroscience-Psychology Program \\ (a) Washington University in St. Louis \\ Campus Box 1073 \\ One Brookings Drive \\ St. Louis, MO 63130-4899 \\ ashriver@wustl.edu \\ 314-882-6955 \\ and \\ Postdoctoral Fellow \\ Rotman Institute of Philosophy and the \\ Mind Brain Institute \\ (a) University of Western Ontario
}

Thanks to John Doris, Julia Driver, Carl Craver, Anna Alexandrova, Dan Habyron, Tom Buller, and an anonymous reviewer for insightful comments on various stages of this paper. I also received helpful feedback at the International Conference on Wellbeing and Public Policy in Wellington, New Zealand and the Minding Animals Conference in Utrecht, the Netherlands. 


\section{Introduction}

Utilitarianism, the ethical doctrine that holds in its most basic form that right actions are those that maximize pleasure and minimize pain, has been at the center of many of the ethical debates around animal welfare. The most well-known utilitarian of our time, Peter Singer, is widely credited with having sparked the animal welfare movement of the past $35+$ years, using utilitarian reasoning to argue against using animals in invasive research that we aren't willing to perform on humans. Yet many people who have argued for the use of animals in invasive experimentation have also appealed to utilitarian ideas by claiming that insofar as lab animals suffer, the suffering is justified by greater benefits produced via the knowledge gained from research. In this paper, I will examine whether the classical utilitarian prescriptions "maximize pleasure" and "minimize pain" should be treated as equals by the theory and, if not, what the possible implications are for research involving nonhuman animals.

The idea that pain has a stronger influence than pleasure is accepted in much of the recent psychology literature on well-being. Some philosophers have also argued that minimizing suffering should play a more important role in ethical theorizing than maximizing pleasure ${ }^{12}$. However, I will argue that neuroscience is uniquely positioned to provide definitive evidence that pleasure and pain are not merely two symmetrical poles of a single scale of experience, but in fact two different types of experiences altogether with dramatically different contributions to our wellbeing. I consider several different conceptions of symmetry, and argue that each is at odds with the most recent empirical results. 


\section{Epicurean Symmetry}

Epicureans famously claimed that the greatest possible pleasure was nothing more than the complete absence of pain ${ }^{3}$. On this view, not only is there a finite ratio between units of pleasure and pain, but units of pleasure can be thought of as literally being units of "not pain." This view, which I will refer to as Epicurean Symmetry, can be defined as follows:

Epicurean Symmetry: Pleasure just is the absence of pain, so decreasing pain increases pleasure, and vice versa.

What does it mean to say that pleasure is merely the absence of pain? A neurophysiological interpretation of Epicurean Symmetry, where pleasure and pain are simply different possible states of the same basic brain areas, has at least some initial plausibility since increased neural activity is observed in similar brain regions during

pleasure and pain. Along these lines, a recent review paper by Lecknes and Tracey ${ }^{4}$ lists sixteen different regions in the brain that have been shown to be activated both during imaging experiments involving pleasure and those involving pain.

However, a general limitation of imaging studies is that they do not distinguish between excitatory and inhibitory neural activity and a particular concern in this case is that changes in activation levels don't tell us how important the various brain regions' activation levels are for pleasure and pain. If we use a stronger criterion of neural activity that is necessary and/or sufficient for the given experiences, the core brain areas involved in pleasure and pain come apart.

At least two functionally and anatomically distinct pathways appear to mediate the experience of pain ${ }^{567}$. Activity in the "sensory pain pathway" appears to play a 
crucial role in the representations of the location of particular pains, of the type of pain (whether it's a "burning" or "cutting" pain, etc.), and of the intensity of the pain. Activity in the "affective pain pathway," on the other hand, mediates the unpleasantness of pain, or how much a pain bothers the subject. Thus, people given morphine (which appears to have a stronger effect on the affective pathway due to a larger number of opiate receptors throughout that pathway) will often report that they still feel pain but that it no longer bothers them as much.

Like the effects of morphine, stopping activity in two particular brain regions via lesions selectively diminishes the unpleasantness of the pain experience. Lesions to select areas in the anterior cingulate cortex or connecting fibers in the cingulum bundle result in patients who say that they still feel pain but that it "is no longer bothersome," Likewise, lesions to the insula cortex result in a condition known as pain asymbolia, where people report feeling pain but show no aversive reaction to painful stimuli ${ }^{9}$. Johansen and Fields ${ }^{10}$ also demonstrated that increasing excitatory neurotransmitters into the anterior cingulate caused rats to avoid locations even in the absence of noxious stimulation, while injecting inhibitory neurotransmitters would cause rats to fail to avoid locations even if they were subjected to noxious stimulation there. And Dong et al. ${ }^{11}$ found that lesioning an area with heavy inputs to the insula led to monkeys who could correctly identify pain but no longer attempted to avoid it. Thus, activity in the anterior cingulate and insula appear to play a crucial role in the experience of the unpleasantness of pain.

On the other hand, Berridge and colleagues ${ }^{12}$ have identified several "hedonic hotspots" associated with pleasure. They define hotspots as areas where activation in the 
area leads to enhanced pleasure reactions. On this definition, the nucleus accumbens, the parabrachial nucleus in the pons, and ventral pallidum count as pleasure hotspots since their activation appears to cause increased pleasure sensations. Out of these three hotspots where activation is sufficient to enhance pleasure sensations, only the ventral pallidum also seems to be necessary for pleasure, as damage to this area leads to ahedonia, a condition where people (and other animals) are unable to experience pleasure $^{1314}$. Thus, a strict neurophysiological interpretation of Epicurean Symmetry appears to be false, since different brain regions are involved in the two experiences.

Perhaps, however, the neurophysiological interpretation is not a particularly interesting version of Epicurean Symmetry. After all, pleasure and pain might play functionally complimentary roles in our psychology even if they rely on different neural architecture. With this in mind, we might alternatively interpret Epicurean Symmetry as a functional claim that pleasure and pain play directly opposing roles in our psychology.

However, this too seems to be at odds with the evidence. In a recent review of a wide range of findings in psychology (not exclusively focused on pleasure and pain), Baumeister et al. concluded, "Taken together, these findings suggest that bad is stronger than good, as a general principle across a broad range of psychological phenomena,"15. Among the examples cited in Baumeister's review were findings that:

- The amount of negative interactions between partners that occurred was far more predictive of marital success than the amount of positive interactions.

- Stronger event related potentials (ERPs) were observed in the brain for subjects reading about people with positive traits performing negative actions than subjects reading about people with negative traits performing positive actions. 
- Bad environments have a powerful effect on the development of children; but good environments have only a very small effect.

- The presence of negative social conflict was more likely to cause mental health issues than the absence of supportive social relations.

- People spend more time thinking about bad events that happened to them than good events.

- Pessimism is a better predictor of health outcomes than optimism.

Thus, across a wide range of domains, negative emotions tend to have a stronger influence than do positive emotions.

Not only does "the bad" exert a stronger effect on our psychology than "the good," but it often produces effects that aren't seen at all with positive stimulation. An experiment by Hietan $\&$ Korpela ${ }^{16}$ indicated that the presentation of negative environmental scenes resulted in an automatic evaluative reaction in subjects while positive scenes did not. Subjects were shown pictures of scenes with High, Medium, and Low degrees of "restorativeness" (which corresponded to pleasant, neutral, and unpleasant conditions). Afterwards, they were shown photos of people making emotional facial expressions and asked to identify the correct emotion. People primed with unpleasant scenes were able to more rapidly identify angry faces after $200 \mathrm{~ms}$, but the positive scenes had no similar effect (though both positive and negative scenes had an effect after $1000 \mathrm{~ms})$. This, the researchers suggested, would fit hypotheses that there could be a distinct survival advantage associated with being able to rapidly and automatically respond to negative circumstances. 
In other words, negative responses to potentially threatening or harmful stimuli appear to play an altogether different role in our psychology than our responses to positive events, which is intuitively plausible given that reactions to negative stimuli helped protect our evolutionary ancestors from potentially life-threatening events. So the Epicurean notion that pleasure is nothing more than the absence of pain is incorrect.

\section{Benthamic Symmetry}

One need not view pleasure as equivalent to the absence of pain to nevertheless be committed to the idea of symmetry between positive and negative experiences in relation to well-being. For example, Jeremy Bentham said both that Nature has placed mankind under the governance of two sovereign masters, pain and pleasure ${ }^{17}$, and also that pleasure "came to the same thing" as immunity from pain ${ }^{18}$

A common idea that fits with Bentham's statements is that of a continuum of experience with a "zero point" that represents "neutral experience." Increasing intensities or durations of pleasure drive a person towards one end of the continuum; increasing intensities or durations of pain towards the other. Thus we can say increasing pleasure "comes to the same thing" as removing pain because both move one's well-being in the same direction along the continuum.

Bentham's claim that pleasure "comes to the same thing" as the absence of pain along with the backdrop of a bipolar scale of how well (or poorly) one's life is going suggests a different notion of symmetry than that of Epicurus. On this view, we might say the following: one unit of pleasure is as good as one unit of pain is bad. Or, to be more precise: 
Benthamic Symmetry: One unit of enjoyment increases our well-being as much as a unit of suffering decreases our well-being, and vice versa.

Once again, I think the empirical evidence speaks against the most intuitive way of understanding this view. Research has consistently shown that negative affect has a much greater influence on subjective reports of well-being than does positive affect. As Larsen and Prizmac put it, "there appears to be a gain function built into the [negative affect] system such that this system produces a larger response, per unit input, than the [positive affect] system,",19.

As evidence for this suggestion, $\operatorname{Larsen}{ }^{20}$ had subjects record their experienced positive and negative affect as well as listing emotions experienced over the day for 56 days and also had raters assign values based on an assessment of how good or bad those events would be for the average person. On days where the objective rankings of events had similar numbers of positive and negative event ratings, it was found that self-reported negative affect was significantly higher than positive affect. Thus, negative affect appeared to more strongly influence subjects' reported well-being.

Several researchers have attempted to more precisely quantify the different influence negative and positive affect have on well-being. The general finding is that people seem to require substantially more positive affect than negative affect in order to exhibit normal functioning. For example, a roughly 5 to 1 ratio of positive to negative affect seems to be required for marriages to last and for both partners to be satisfied ${ }^{2122}$. Fredrickson and Losada ${ }^{23}$ took up more explicit measurements of the ratio between positive and negative affect in "mentally flourishing individuals" and found that substantially higher positive than negative affect was required for mental flourishing. 
Analysis of business teams found that a 2.9 positive to negative affect ratio was the rough cutoff dividing successful from unsuccessful teams, with success being defined in terms of profitability, customer satisfaction, and evaluations by others. And in two other data sets, flourishing college students had ratios of 3.2 to 1 and 3.4 to 1 positive to negative affect, while non-flourishing students had ratios of 2.3 to 1 and 2.1 to 1 . In this case, flourishing was defined as exhibiting positive functioning in 6 out of the 11 categories $^{24}$. From these results, as a rough approximation (due to the wide variety in the types of measurements), Larsen and Prizmic concluded that the "degree to which bad is stronger than good" can be approximated by the equation: Positive Affect $* 3.14=$ Negative Affect $^{25}$. Similarly, Fredrickson and Losada argued that 2.9 positive to negative affect ratio was needed for mental flourishing ${ }^{26}$.

Thus it seems that the most intuitive way of thinking about Benthamic Symmetry is false. Equal amounts of positive and negative experiences do not balance out to neutrality; in fact, negative experience has "more weight" and drags one down faster than positive experience picks one $\mathrm{up}^{27}$. A straightforward claim that units of enjoyment are weighted equally to units of suffering in relation to well-being is simply false.

However, an important point by James Griffin ${ }^{28}$ looms in the background. Griffin suggested that if units of pain mattered more than units of pleasure we could simply think of them as contributing differently to a scale of well-being. Thus, we might think that three units of pleasure only equal one unit of pain on a scale of well-being, but nevertheless retain the basic symmetry captured in Bentham's claim. Or, to put it a different way, we might claim, "what we thought was three units of positive affect was 
actually only one unit of positive affect, and we simply need to adjust our scales accordingly."

This claim, however, requires additional empirical support, as it suggests there is some function that can tell us how positive affect relates to negative affect in measurements of well-being. In the absence of such support, this revised Benthamic Symmetry should be accepted only if we already have good intuitive reasons to think that positive and negative affect contribute similarly to our well-being. However, in the final set of evidence I present, I hope to show that this is precisely what we lack. Digging further into the neuroscience, I argue that positive and negative affect are not similarly related to our well-being because they relate to our desire and reward systems in fundamentally different ways.

\section{Sidgwickian Symmetry}

Henry Sidgwick seemed to endorse a Benthamic notion of Symmetry. He defined the Greatest Happiness as:

The greatest possible surplus of pleasure over pain, the pain being conceived as balanced against an equal amount of pleasure so that the two contrasted amounts annihilate each other for purposes of ethical calculation ${ }^{29}$.

However, one of Sidgwick's statements also suggests a different notion of symmetry: Pain may be regarded as the negative quantity of pleasure, and that accordingly any statements made with respect to pleasure may be at once applied, by obvious changes of phrase, to pain ${ }^{30}$.

Sidgwick used his statement in a narrow context, where it could be thought to come to virtually the same thing as Benthamic Symmetry. However, we can broaden the scope to 
locate an implicit assumption that seems to lurk behind many discussions of well-being and utility. If we accept a bipolar scale of well-being and ill-being, the second part of Sidgwick's statement might be thought to suggest something like this:

Sidgwickean Symmetry: If a claim describing the relationship between enjoyment and well-being is true, an otherwise identical statement substituting "suffering" for "enjoyment" and "ill-being" for "well-being" must also be true.

If this statement is correct, then we would seem to have at least some justification for treating pleasure and pain as symmetrical. Even if we have to adjust the units of the utility scales, the same basic relationships hold between the two types of experiences and utility, albeit in different directions.

To take on this claim, I begin with a consideration of the two types of experiences. When we suffer, we necessarily find the suffering unpleasant. If an experience, such as pain, lacks unpleasantness, then it isn't an instance of suffering. In other words, there is a necessary connection between suffering and the unpleasantness of an experience. Furthermore, there is a necessary connection (given our psychology) between suffering and having a desire for the suffering to end: one cannot be suffering without having some desire for the suffering to end. This desire isn't necessarily overriding; that is, one might have a desire for suffering to cease but have a stronger desire to not tell the terrorists where one's fellow troops are located, but nevertheless the desire is intrinsically connected to the experience of suffering. Thus, there is an intrinsic connection between the experience of suffering and a desire for the suffering to cease.

On the other hand, I claim no such intrinsic connection exists between enjoyment and desire. It's true that many people greatly desire certain pleasures and that most, 
while in a state of pleasure, have a strong desire for the pleasure to continue. However, I will argue, this connection is contingent, dependent on individual variations in how much different people value different pleasures, and in how much one person values pleasure at different times. Sometimes we experience intense pleasures without having a desire for the pleasure to continue. Sometimes we have very strong desires but experience very little pleasure when those desires are satisfied. Pleasure and desire come apart. Thus suffering, I claim, has a more direct connection to our utility than does pleasure.

I believe this claim, strange as it may sound, is supported by recent findings in the study of the neuroscience of reward ${ }^{31}$. In particular, a large body of research has shown that pleasure can come apart from the desire and reward pathways in the brain. First, consider research from Lamb et al. ${ }^{32}$ where subjects were given small traces of addictive drugs. Subjects preferentially chose solutions that contained morphine over saline solutions even though they reported experiencing no additional feelings of pleasure. In a similar study by Fischman \& Foltin ${ }^{33}$, subjects were willing to preferentially work for solutions containing drugs despite reporting no pleasure from the drugs. Thus, no conscious experience of pleasure was driving their motivation. Second, rats with lesions to the motivational system continued to make characteristic facial expressions of pleasure when given sucrose solution, though they were no longer motivated to consume the sucrose $^{34}$. Finally, research has shown that rats can be stimulated to vigorously engage in behavior even though that behavior is apparently no longer pleasant or perhaps even unpleasant for the rats. In one study ${ }^{35}$, amphetamines were administered that caused the rat to frantically push a lever that released sugar pellets. However, testing revealed that the rats did not find the pellets any more pleasurable. Similarly, a different experimental 
manipulation stimulated the motivational system to get rats to eagerly eat large amounts of food. However, the facial expressions of the rats as they were eating indicated that they actually disliked the taste of the food despite continuing to eat. The combined implication of all of these results is that the pleasure and desire systems are distinct or at least dissociable.

On the other hand, researchers have had less luck conclusively identifying the "punishment" centers in the brain, but I believe that those areas associated with the affective dimension of pain are good candidates. Recall that rats with lesions to the ACC no longer appear to have a desire to avoid noxious stimuli even though they show normal immediate withdrawal behavior. And as noted above, excitatory injections into the ACC will cause rats to desire to avoid locations even in the absence of noxious stimulation while inhibitory injections to the ACC will prevent rats from avoiding locations even in the presence of noxious stimulation. Additionally, lesioning an area closely connected to the insula resulted in monkeys who could correctly identify normally painful stimulation but no longer attempted to avoid it. Thus, the ACC and insula appear to not only be associated with the unpleasantness of pain, they also are associated directly with the desire to avoid the pain. In other words, suffering cannot be pulled apart from desire in the way that pleasure can.

Our understanding of the neuroscience of pleasure and pain is still in early stages, and I concede that future results could undermine this suggestion. However, if it is true, as I suggest, that pleasure is intrinsically separable from appetitive desire while suffering is intrinsically connected with aversive desire, I think this counts as strong evidence that pleasure and pain are not just two different experiences, but in fact are two wholly 
different types of experiences. Thus, even if one believes that both pleasure and pain matter for well-being, it is a mistake to assume, without argument, that they matter in the same way. And even if neuroscience shows that my distinction is ultimately incorrect, I hope this account at least demonstrates how the neuroscience of pleasure and pain is relevant to discussions of utility. Given the large body of evidence suggesting an asymmetry, ethicists cannot simply assume that what is said about pleasure has similar implications for pain, and vice versa.

\section{Implications for Animal Welfare:}

Even if one does think pleasure and pain relate to utility asymmetrically, this doesn't tell us much about the precise relations. One possibility, made more palatable by the idea that they're two different types of experiences, is that pleasure and pain might in fact be incommensurable values; values, that is, not measurable on a single scale. On this view, one might think that there's no coherent way of "trading" one type of value for another.

This view seems implausible in relation to our own lives. People frequently choose to endure some pains or discomforts in order to achieve future pleasures, and likewise choose to forgo certain pleasures in order to avoid future pains. On the other hand, sometimes people decide that they're having so much fun that tomorrow's hangover might actually be worth it. Thus, we appear to constantly be weighing the value of various pleasures and pain in our decisions, and the idea that they are radically incommensurable doesn't fit with everyday experience. 
However, just because intrapersonal comparisons can be easily made, this doesn't necessarily mean interpersonal comparisons will be similarly straightforward. In fact, if, as I have suggested, it turns out that pleasure is only contingently related to our desires, this raises certain puzzles about how we should compare the value of pleasures between different people. Let's say person A has a strong desire for pleasure and person B has only a weak desire for the same amount of pleasure. Does this mean that a pleasure of that intensity matters more from an ethical point of view if experienced by A rather than B? Or what if person C gets a lot of pleasure out of eating cake but doesn't have a strong desire to eat a slice, whereas person D has a strong desire for the cake but doesn't get much pleasure from eating it? It's difficult to say with certainty whose eating the cake has more utility in this situation.

However, I think similar problems do not arise for the evaluation of suffering since, I have argued, that the degree of unpleasantness is correlated with the desire to avoid the experience. We can compare suffering between individuals because the strength of the experience tracks the strength of the desire to avoid that experience. Since suffering has a fixed amount of disutility, but the utility of pleasure is variable, I think the utilitarian prescription to "minimize suffering" makes a more direct moral demand on us than does "maximize pleasure."

Furthermore, if suffering and pleasure really are different types of experiences, we might need to rethink some of the basic maneuvers made in utilitarian arguments. On the asymmetrical view, we would still have moral reason to choose actions that cause some suffering for the sake of avoiding a greater quantity of suffering. Yet we might not, even in principle, be justified in choosing actions that cause suffering in one individual 
for the sake of moving a large number of people from a state of mild happiness to a state of intense happiness, no matter intense the pleasurable experience or how large the group of people.

This idea already seems familiar in utilitarian discussions of animal welfare. In essence, the argument against eating factory-farmed meat is that the pleasure of eating meat rather than a vegetarian diet, viewed impartially, cannot justify the amount of suffering caused to the animal in the factory farming process. Typically, the thought is that the two states are comparable but that the intensity of the pain far outweighs the intensity of the pleasure. The difference between the standard account and the asymmetrical view, however, is that on the latter view one might think that no amount of pleasure for one person, or indeed even many people, (where that pleasure is not itself preventing suffering) could even in principle justify causing intense suffering in another being. As I have argued elsewhere ${ }^{36}$, there's good reason to think that at the very least all mammals suffer similarly to humans. Suggestions that mammals might exhibit "pain-like behavior" without suffering are implausible in the face of evidence that similar sensory/affective pain dissociations can be found in mammals, with the affective pain behaviors mediated by the same regions that underlie suffering in humans ${ }^{373839}$.

Asymmetric utilitarianism draws sharper distinctions than typical utilitarian calculations, since it rules out certain types of trades. However, the implications are still more complicated than they might seem. To examine this further, I consider three types of cases of animal research using utilitarian reasoning with an asymmetrical emphasis on preventing suffering. 
First, consider research on animals designed to study the neurobiology that leads to negative experiences in humans, such as pain, anxiety, or suffering, or to study health conditions that could lead to suffering. Since this research is premised, in most cases, on hopes of being better able to alleviate such suffering in the future, the research could be justified if it leads to a reasonable hope that causing a small amount of suffering in the lab animals would lead to a greater prevention of suffering for a large number of people (and possibly other animals) in the future. Thus, causing suffering to prevent greater amounts of future suffering can be supported on an asymmetrical conception of utilitarianism.

On the other hand, a second type of experiments, those that cause suffering in animals for the sake of understanding positive experiences like gustatory pleasure, the joy of music, etc., might initially be thought to be ruled out by an asymmetrical conception of utility, since increasing future pleasures could not, even in principle, be worth the cost of causing suffering. However, defects in the pleasure and reward system can lead to miserable existences as cases of severe depression indicate, and it remains to be seen how much it is the case that some amount of positive affect is required in order to prevent one from experiencing negative affect. Thus, one can provide a utilitarian argument for animal research in service of the study of pleasure even on the asymmetric view since a certain amount of pleasure seems to be necessary to avoid suffering.

However, for a third type of experiment involving animals, those involving testing for the sake of new leisure products (such as Singer's example of $\operatorname{cosmetics}^{40}$ ), the asymmetrical view could quite strongly condemn the use of animals experiments, assuming (quite plausibly) that these products only provide extremely minimal increases 
in happiness unlikely to prevent suffering in any meaningful way. Examples such as testing for cosmetic products have already been condemned by most utilitarians writing about animal welfare on the grounds that the minimal amounts of happiness created by new products would not outweigh the suffering caused to animals. However, on the traditional utilitarian view, it is necessarily the case that some amount of small increases in pleasure adding up would eventually outweigh the disvalue of the suffering caused. In other words, if enough people got minor pleasure from a new makeup product, then causing suffering to some finite number of animals would be justified. However, on the asymmetrical view, this conclusion would no longer follow. Since suffering is asymmetrically more important than suffering, no number of tiny pleasures could ever, even in principle, justify the causing of suffering in human or nonhuman animals.

Thus, I think, the asymmetry view fits well with many utilitarian intuitions about animal welfare. Animal research for trivial purposes is strictly ruled out if that research causes suffering. However, animal research for the prevention of disease and disorder and general knowledge that might indirectly prevent suffering in the future remains a complicated, but in principle justifiable, practice.

So this empirically-informed asymmetric account of utility offers a new way of evaluating certain tradeoffs in animal welfare debates, though it preserves many common utilitarian intuitions about outcomes. Ultimately, even if future research suggests the details of my account are mistaken, I hope to have made clear that any ethical theory that involves weighing the positive and negative experiences associated with different outcomes will need to pay close attention to the empirical results associated with various types of positive and negative experiences. The nature of pleasure and pain in humans 
and in other animals is determined, at least in part, at the neural level, and ethicists should take pains to make sure that their assumptions about pleasure and pain are supported by the facts. 


\section{Notes}

${ }^{1}$ Mayerfeld J. Suffering and Moral Responsibility New York: Oxford University Press; 2002.

${ }^{2}$ Popper K. The Open Society and Its Enemies Princeton, NJ: Princeton University Press; 1952.

${ }^{3}$ Rackham H, ed. Cicero: De Finebus Bonorum et Malorum Cambridge: Harvard University Press; 1983.

${ }^{4}$ Lecknes S, Tracy I. A common neurobiology for pain and pleasure. Nature Reviews Neuroscience 2008;9:314-20.

${ }^{5}$ Rainville P, Duncan GH, Price DD, Carrier B, Bushnell MC. Pain affect encoded in human anterior cingulate but not somatosensory cortex. Science 1997;277:968-71.

${ }^{6}$ Price DD. Psychological and neural mechanisms of the affective dimension of pain. Science 2000;288:1769-72.

${ }^{7}$ Vogt B, Sikes RW. Cingulate nociceptive circuitry and roles in pain processing: the cingulate premotor pain model. In: Vogt B, ed. Cingulate Neurobiology and Disease.

New York: Oxford University Press; 2009; 311-338.

${ }^{8}$ Foltz EL, White LE. Pain relief by frontal cingulotomy. Journal of

Neurosurgery 1962;19:89-100.

${ }^{9}$ Berthier M, Starkstein S, Leiguards R. Asymbolia forp: a sensory-limbic disconnection syndrome. Annals of Neurology 1988;24:41-9.

${ }^{10}$ Johansen JP, Fields HL. Glutamatergic activation of anterior cingulate cortex produces an aversive teaching signal. Nature Neuroscience 2004;7(4):398-403.

${ }^{11}$ Dong WK, Hayashi T, Roberts VJ, Fusco BM, Chudler EH. Behavioral outcome of posterior parietal cortex injury in the monkey. Pain 1996;64:579-587.

12 Smith KS, Mahler SV, Pecina S, Berridge KC Hedonic hotspots: generating sensory pleasure in the brain. In: Kringlebach ML, Berridge KC, eds. Pleasures of the Brain.

New York: Oxford University Press; 2010:27-49.

${ }^{13}$ Miller JM, Vorel SR, Tranguch AJ, Kenny ET, Mazzoni P, van Gorp WG et al. Anhedonia after selective bilateral lesion of the globus pallidus. American Journal of Psychiatry 2006;163:786-788.

${ }^{14}$ Cromwell HC, Berridge KC. Where does damage lead to enhanced food aversion: the ventral palidum/substantia innominata or lateral hypothalmus? Brain Research 1993;624:1-10.

${ }^{15}$ Baumeister RF, Bratslavsky E, Finkenauer C, Vohs KD. Bad is stronger than good. Review of General Psychology 2001;5:323-370.

${ }^{16}$ Hietanen JK, Korpela KM. Do both negative and positive environmental scenes elicit rapid affective processing? Environment and Behavior 2004;36:558-77.

${ }^{17}$ Bentham J. Introduction to the Principles of Morals and Legislation New York: Oxford University Press ;1789:11.

${ }^{18}$ See note 17, Bentham 1789:34.

${ }^{19}$ Larsen RJ, Prizmic Z. Regulation of emotional well-being. In: Eid M, Larsen R, eds. The Science of Subjective Well-Being New York: Guilford Publications; 2008:261.

${ }^{20}$ Larson, RJ. Differential contributions of positive and negative affect to subjective well being. In: Da Silva JA, Matsushima EH, Riberio-Filho NP, eds. Annual Meeting of the 
International Society for Psychophysics. Rio de Janeiro, Brazil: Editora Legis Summa Ltda; 2002; 18:186-90..

${ }^{21}$ Gottman JM. What Predicts Divorce? The relationship between marital process and marital outcomes. Hillsdale, NJ:Lawrence Erlbaum Associates;1994.

${ }^{22}$ More precisely, the cutoff was 5.1 to 1 for verbal content and 4.7 to 1 for emotional expressions for "flourishing" marriages.

${ }^{23}$ Fredrickson BL, Losada MF. Positive affect and the complex dynamics of human flourishing. American Psychologist 2005;60:678-86.

${ }^{24}$ The categories were self-acceptance, purpose in life, environmental mastery, positive relations with others, personal growth, autonomy, social coherence, social integration, social acceptance, social contribution, and social actualization.

${ }^{25}$ See note 19, Larsen, Prizmic 2008:258-89.

${ }^{26}$ Fredrickson BL, Losada MF. Positive affect and the complex dynamics of human flourishing. American Psychologist 2005;60:678-86.

${ }^{27}$ For many people, notably those privileged enough to be in academia, this depressing fact is neutralized by the fact that we have a much greater quantity of mildly pleasant experiences than we do negative experiences, and indeed the general "set point" for most people in normal situations is a mildly pleasant experience.

${ }^{28}$ Griffin J. Is unhappiness more important than happiness? Philosophical Quarterly 1979;114:47-55.

${ }^{29}$ Sidgwick H. The Methods of Ethics Indianapolis: Hacket Publishing Company 1907:129.

${ }^{30}$ See note 29, Sidgwick 1907:125.

${ }^{31}$ Much of the following paragraph is based on Tim Schroeder's helpful discussion in Schoeder, T. The Three Faces of Desire New York: Oxford University Press; 2004.

${ }^{32}$ Lamb RJ, Preston KL, Schindler CW, Meisch RA, Davis F, Katz JL et al. The reinforcing and subjective effects of morphine in post-addicts: a dose-response study. Journal of Pharmacology and Experimental Therapies 1991;259:1165-73.

${ }^{33}$ Fischman MW, Foltin RW. Self-administration of cocaine by humans: a laborator perspective. In: Bock GR, Whelan J, eds. Cocaine: Scientific and Social Dimensions Chicester, England: Wiley; 1992;165- 80.

${ }^{34}$ Berridge K, Robinson TE. What is the role of dopamine in reward: hedonic impact, reward learning, or incentive salience? Brain Research Reviews 1998;28:309-69.

${ }^{35}$ Wyvell C, Berridge K. Intra-accumbens amphetamine increases the conditioned incentive salience of sucrose reward: enhancement of reward 'wanting' without enhanced 'liking' or response reinforcement. The Journal of Neuroscience 2000;20:8122-30.

${ }^{36}$ Shriver A. Minding mammals. Philosophical Psychology 2006;19:433-42.

${ }^{37}$ Allen C, Fuchs P, Shriver A, Wilson H. 2005. Deciphering animal pain. In: Aydede M ed. Pain: new essays on the nature of pain and the methodology of its study Cambridge: MIT Press; 2005:352- 366.

${ }^{38}$ LaGraize S, Labuda, C, Rutledge R, Jackson R, Fuchs P. Differential effect of anterior cingulated cortex lesion on mechanical hypersensitivity and escape/avoidance behavior in an animal model of neuropathic pain. Experimental Neurology 2004;188, 139-148. 
${ }^{39}$ LaGraize S, Borzan J, Peng YB, Fuchs P. Selective regulation of pain affect following activation of the opiod anterior cingulate cortex system. Experimental Neurology 2006; 197:22-30.

${ }^{40}$ Singer P. Practical Ethics New York: Cambridge University Press; 1993. 